\title{
Research on the Impact of Key Equipment Failure on Subway Station Capacity
}

\author{
Sai $\mathrm{Li}^{1, \text { a }}$, Xiukun Wei ${ }^{2, \mathrm{~b},{ }^{*}}$ \\ ${ }^{1}$ Beijing Jiaotong University, Beijing 100044, China; \\ ${ }^{2}$ Beijing Jiaotong University, Beijing 100044, China. \\ a17120965@bjtu.edu.cn, b, ${ }^{\star}$ xkwei@bjtu.edu.cn
}

\begin{abstract}
Under the complex passenger flow environment, the failure of key equipment will affect the subway station capacity (SSC), and the average travel time of passengers is considered as the evaluation indicator of subway station capacity. The key equipment capacity model and station capacity model considering key equipment failure are established using the idea of binomial distribution and mathematical expectation. Besides, Xinjiekou station in Beijing subway is taken as a case, and passenger flow simulation model based on AnyLogic is established. Finally, this paper analyzes the impact of key equipment failures on the average travel time of passengers on the base of passenger flow simulation model, and gives corresponding maintenance recommendations.
\end{abstract}

Keywords: Subway station capacity, key equipment failure, binomial distribution, AnyLogic.

\section{Introduction}

With the advantages of safety, convenience and punctuality, urban rail transit has been favored by travelers and has become an indispensable mode of transportation in many cities. Under complex passenger flow environment, electromechanical equipment such as gates and escalators at subway station will fail after being put into use, which will affect the capacity and efficiency of the station.

In order to quantitatively analyze the impact of key equipment failure on the capacity and efficiency of the station, a passenger flow simulation model for subway station needs to be established. Simulation models are suitable for describing station in different perspectives, but few consider the capacity calculation problem. For example, a modelling approach based on discrete-event simulation and response surface methodology (RSM) dealt with average passenger travel time optimization problem inherent to the metro planning process (2009). A queuing network model of station is created based on $\mathrm{M} / \mathrm{G} / \mathrm{C} / \mathrm{C}$ state dependent queuing network and DTMC by Xu (2014). These studies focused on how to model the station, evaluate the usage of equipment or level of service (LOS), but existing literatures could not provide a method to calculate capacity integrating simulation models considering key equipment failures.

This paper will build passenger flow simulation model and station capacity model considering key equipment failures and then analyze the impact of key equipment failures on the average travel time of passengers and give corresponding maintenance recommendations.

\section{Subway Station Capacity and Gathering and Scattering Efficiency}

\subsection{Definition of SSC.}

According to the gathering and scattering process, the subway station can be regarded as a service system referring to the idea of queuing theory, as shown in Figure 1. The input is the number of passengers entering the station system per unit time, the output is the number of passengers leaving the station system within the unit time. The system service time includes the passenger's arrival time, departure time, and waiting time on the platform. The system service time can reflect the gathering and scattering efficiency of the subway station.

SSC can be defined as the maximum number of passengers that can be served by one station in a given period of time, typically $1 \mathrm{~h}$, under specified operating conditions, without unreasonable delay, hazard, or restriction, and with reasonable certainty. 


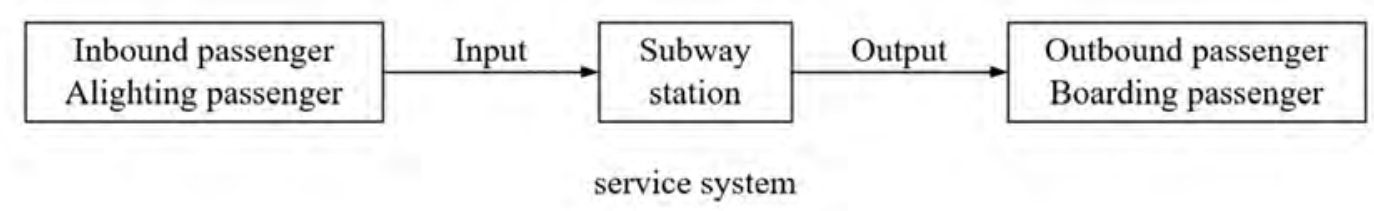

Fig 1. Subway station service system.

\subsection{Evaluation Indicator of SSC.}

Based on previous sections, SSC may be determined by incoming passenger volume, incoming passenger characteristics, timetable, capacity of one single equipment, equipment utility control strategy of the whole station and passenger control strategy of the station and so on.

Because of the number of people served by the station per unit time does not reflect the gathering and scattering efficiency, this paper uses the average travel time of passengers within the station as an evaluation indicator to quantitatively reflect changes of subway station capacity. The average travel time of passengers includes arrival time and departure time. The arrival time is defined as the time when the passenger arrives at the platform from the entrance to the platform, and the departure time is defined as the time when the passenger arrives at the non-payment area of the hall from the platform screen door. The average travel time of passengers can be formulated as follows:

$$
T_{a}=\frac{\sum_{i=1}^{m} t_{i}^{i n}}{m}+\frac{\sum_{j=1}^{n} t_{j}^{\text {out }}}{n}
$$

In which $t_{i}^{\text {in }}$ is the arrival time, $t_{i}^{\text {out }}$ is the departure time.

\section{Station Capacity Model Considering Equipment Failure}

\subsection{Classification of Subway Station Facilities}

Station facilities are an important part of subway station which can be divided into two types: civil engineering facilities and electromechanical equipment. Civil engineering facilities include stairs, walkways, etc. electromechanical equipment includes gates, escalators, and platform screen doors. The reliability of civil engineering facilities is equal to 1 because they will not be damaged for a long period of time after being put into use. Gates, escalators, and platform screen doors will fail, so the reliability is less than 1 . When the equipment fails, it may affect the capacity of the subway station. The reliability of these equipment has a significant impact on the station capacity. Therefore, gates, escalators and screen doors are regarded as the key equipment of subway station.

\subsection{Key Equipment Capacity Model Considering Failure}

The key equipment has two operating states: normal and malfunction. Assume that: the reliability of key equipment such as the gate is $R(t)$; the max capacity of key equipment is $\mathrm{C}$ when the equipment is working properly; the capacity of key equipment is 0 when the equipment fails. At time $\mathrm{t}$, the probability of the equipment working properly is $R(t)$, the expected capacity of single equipment considering failure is $\mathrm{C} * \mathrm{R}(t)$. The equipment status is independent of each other. When the number of the equipment in the equipment group is $n$, related capacity parameters is shown in Table 1 according to the binomial distribution.

Table 1. Capacity parameters of the equipment group.

\begin{tabular}{ccccc}
\hline The number of normal equipment $/ i$ & 0 & 1 & $\ldots$ & $\mathrm{n}$ \\
\hline Probability $/ P_{i}$ & $(1-R(t))^{n}$ & $C_{n}^{1} *(1-R(t))^{n-1 * R(t)}$ & $\ldots$ & $(R(t))^{n}$ \\
the max capacity of the equipment group $/ C_{i}$ & 0 & $\mathrm{C}$ & $\ldots$ & $\mathrm{n} * \mathrm{C}$ \\
\hline
\end{tabular}


The expected capacity of the equipment group considering equipment failure is $\sum_{i=0}^{n}\left(C_{i} * P_{i}\right)$.

\subsection{Station Capacity Model Considering Equipment Failure}

When the key equipment fails, the number of equipment working normally in the station decreases, and subway station capacity may decrease. According to the different functions of key equipment in subway station, it can be divided into inbound gate group, outbound gate group, upward escalator group, downward escalator group, up direction screen door group and down direction screen door group regardless of the equipment layout. Assume that: the reliability of the gate, the escalator, and the screen door is $R_{1}(t), R_{2}(t), R_{3}(t)$.And the number of the inbound gates, the outbound gates, the upward escalators, the downward escalators, the up direction screen doors, the down direction screen doors is $m_{1}, m_{2}, m_{3}, m_{4}, m_{5}, m_{6}$.It can be seen that the subway station has $\mathrm{n}$ different operating states and the corresponding probability, capacity and the average travel time for each operating state is $P_{i}, C_{i}$ and $T_{i}$. Capacity and the average travel time can be calculated by simulation model based on AnyLogic.

The expected capacity $C_{e}$ and the expected average travel time $T_{e}$ considering equipment failure can be formulated as follows:

$$
\begin{gathered}
\mathrm{n}=\prod_{i=1}^{6}\left(m_{i}+1\right) \\
C_{e}=\sum_{i=1}^{n}\left(C_{i} * P_{i}\right) \\
T_{e}=\sum_{i=1}^{n}\left(T_{i} * P_{i}\right)
\end{gathered}
$$

\section{Passenger Flow Simulation Model Based on Anylogic}

Xinjiekou station in Beijing subway is taken as a case study, and passenger flow simulation model based on AnyLogic is established.

\subsection{The General Conditions of Xinjiekou Station}

Xinjiekou station is an underground station of Beijing subway Line 4 . The station hall is a rectangular area with a length of $60 \mathrm{~m}$ and a width of $17 \mathrm{~m}$, and the platform is a rectangular area with a length of $118 \mathrm{~m}$ and a width of $9 \mathrm{~m}$. Streamline layout is shown in Figure 2.
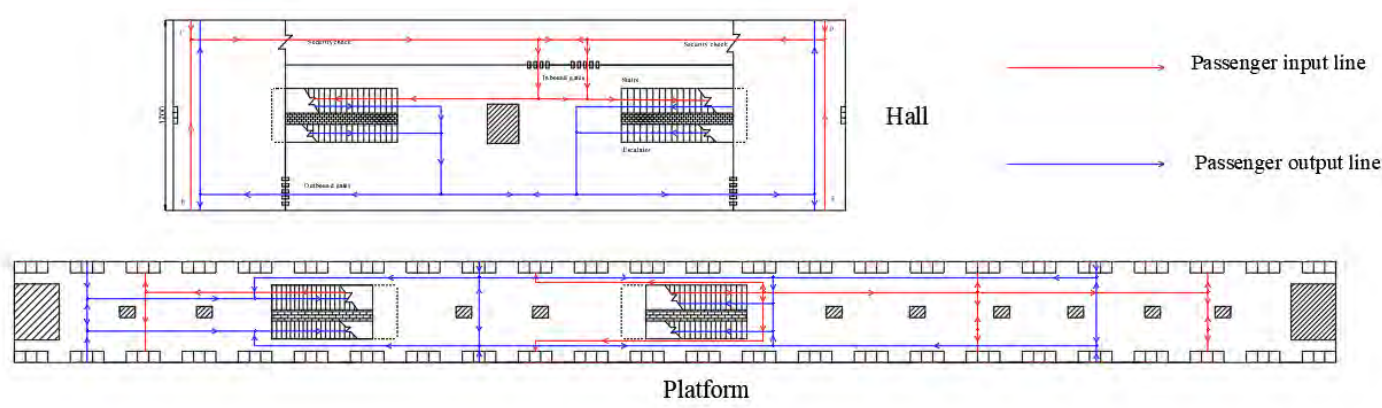

Fig 2. The streamline layout of Xinjiekou station.

\subsection{AnyLogic Model Building}

AnyLogic model include station environment model and pedestrian behavior model. According to the layout of station, the station environment model is established. According to the streamline layout, the pedestrian behavior model is established and shown in Figure 3. and Figure 4. And it is necessary to calibrate the service parameters in the Ped Service block. Through the field research on the station, the related service parameters are shown in table 2 . 


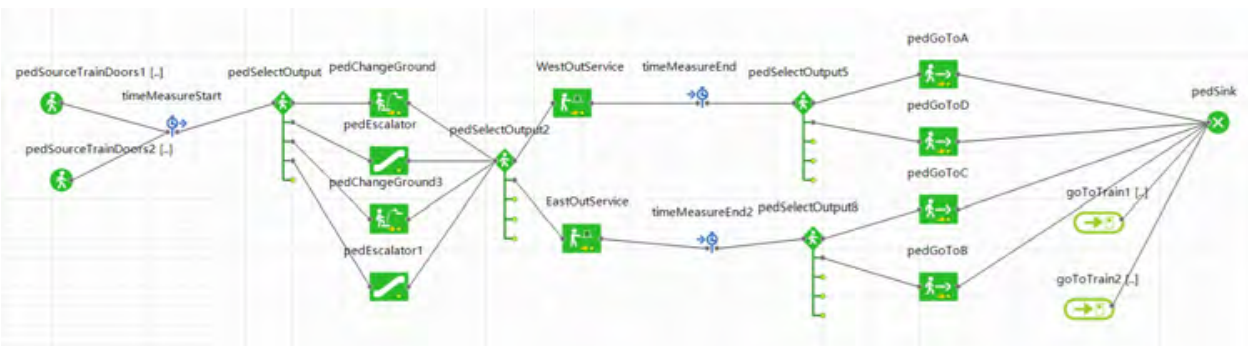

Fig 3. The outbound pedestrian behavior model.

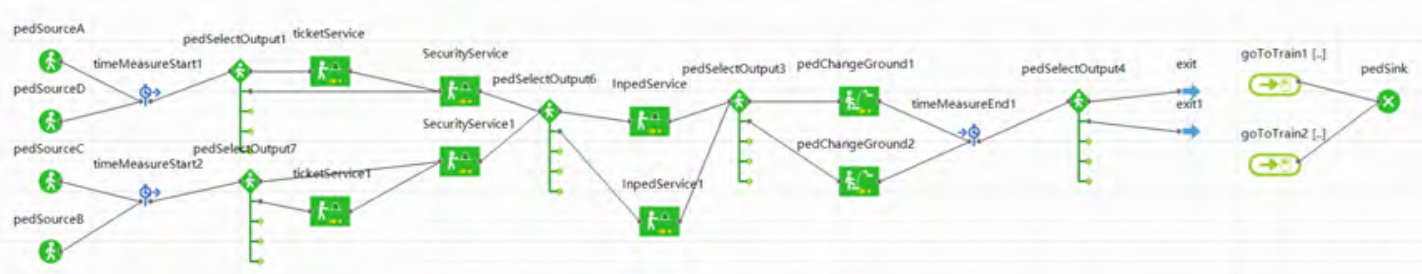

Fig 4. The inbound pedestrian behavior model.

Table 2. Service parameters of the station facilities.

\begin{tabular}{cccc}
\hline Block & Station facilities & Parameter name & Parameter assignment \\
\hline \multirow{3}{*}{ Ped Service } & TVM & Delay time & normal $(12.0,40.0)^{*}$ second \\
& Security check & Delay time & uniform $(3.0,6.0)^{*}$ second \\
& Gate & Delay time & exponential $(3.0) *$ second \\
\hline
\end{tabular}

\subsection{AnyLogic Model Validation}

After building the Anylogic simulation model, the accuracy and reliability of the model can be verified by comparing the simulation results with the actual data.

The subway train serving this station consists of six vehicles. Each vehicle is fitted out with four shared alighting-boarding gates. The headway time and dwell time running by the current timetable are $150 \mathrm{~s}$ and $30 \mathrm{~s}$. From the real video data gathered at platform and from the literature (Cao and Yuan, 2009), it seems that the Normal distribution best fits the number of passengers getting on trains. Passenger routing probabilities were estimated as the ratio of the passengers who arrived at an entrance and followed the specific route, by the total number of passengers who entered the system from this entrance. Other are passenger flow data described in table 3.

Table 3. Passenger flow volume at each entrance.

\begin{tabular}{ccccc}
\hline Entrance & A & B & C & D \\
\hline Passenger flow volume during non-peak hours (person $/ \mathrm{h}$ ) & 400 & 680 & 420 & 580 \\
Passenger flow volume during peak hours (person $/ \mathrm{h}$ ) & 1200 & 1480 & 1160 & 1390 \\
\hline
\end{tabular}

Considering the randomness of Anylogic simulation, it is necessary to conduct multiple simulations experiments and take the average value. During non-peak time, the real departure time is $134 \mathrm{~s}$, the real arrival time is $92 \mathrm{~s}$, the simulation departure time is $130 \mathrm{~s}$, the simulation arrival time is $88 \mathrm{~s}$. During peak time, the real departure time is $187 \mathrm{~s}$, the real arrival time is $155 \mathrm{~s}$, the simulation departure time is $178 \mathrm{~s}$, the simulation arrival time is $148 \mathrm{~s}$.

The error between simulation results and actual data is relatively small, so the accuracy and reliability of the model can be verified. 


\section{Analysis of the Impact of Key Equipment Failure on Subway Station Capacity}

\subsection{The Impact of the Number of Failed Key Equipment on the SSC}

When other equipments are working properly, the impact of the number of failed gate on $T_{a}$ is shown in Figure 5. The two escalators in the station are both upward escalators, so the impact of the number of failed escalator on $T_{a}$ is shown in Figure 6.

It can be seen that the impact of key equipment failure during peak time is much more serious than the impact during non-peak time. Key equipment failure during peak time deserve more attention. During peak time, when the number of inbound gate failures is not more than 3 or the number of outbound gate failures is not more than 2 , the average travel time is almost unchanged. Under the current passenger flow conditions, passengers' travel has not been affected, because the gates are not the capacity bottleneck of the station. When the number of inbound gate failures is more than 3 or the number of outbound gate failures is more than 2, the average travel time increase rapidly and gates need to be repaired in time. So the critical value of inbound gate failures is 3 and the critical value of outbound gate failures is 2 . In addition, the impact of outbound gate failure is much more serious than the impact of inbound gate failure, so the outbound gate failure deserves more attention, and have higher priority for repair.
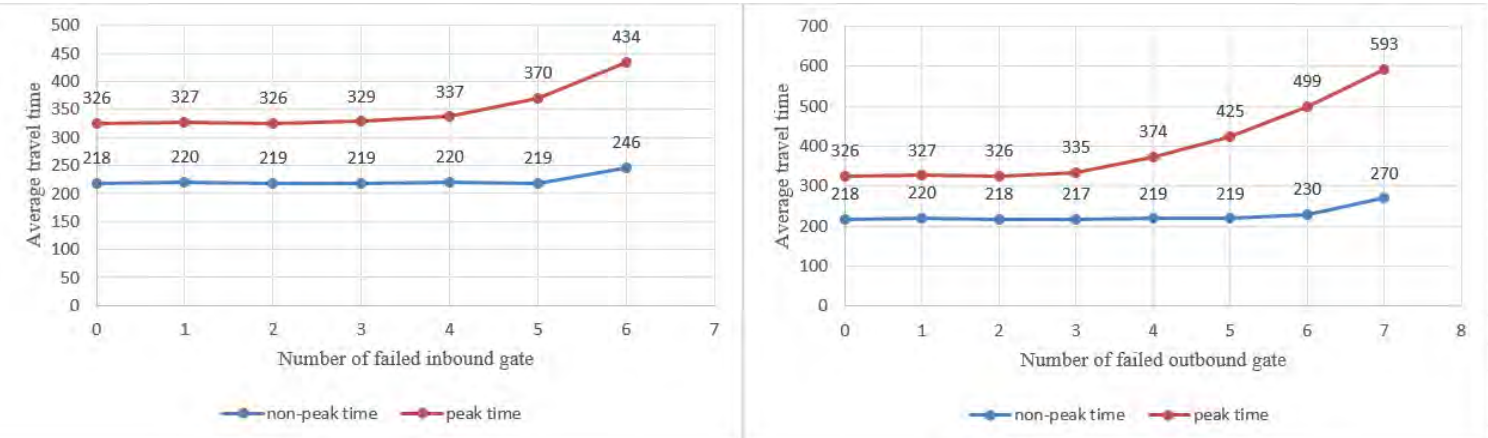

Fig 5. The impact of the number of failed gates on average travel time.

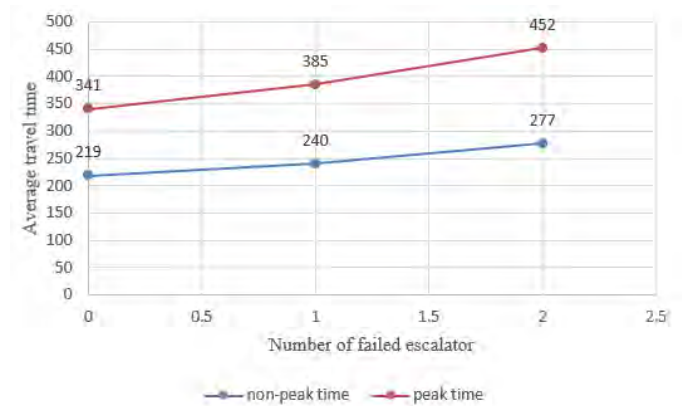

Fig 6. The impact of the number of failed escalators on average travel time.

When the escalator fails, the travel time increases significantly, and with the increase of the number of failures, the average travel time is longer. That's because when the escalator fails, the passenger's route from the platform to the hall is reduced, and the passenger streamline is more conflicted on the stairs. It is necessary to prevent escalator from malfunctioning, especially during rush hour.

\subsection{The Impact of the Reliability of Key Equipment on the Expected Station Capacity}

The impact of the number of key equipment failures on SSC does not take into account the failure probability, and the different number of key equipment failures corresponds to different probability. The station capacity model considering equipment failure is used to study the impact of the reliability of key equipment on the expected station capacity. 
When all inbound gates or outbound gates fail, the travel time is infinite. But in order to measure time, the travel time is assigned to $3600 \mathrm{~s}$. When the reliability of other equipments is 1 , the impact of the reliability of gate on $T_{a}$ is shown in Figure 7. When the reliability of other equipments is 1 , the impact of the reliability of escalator on $T_{a}$ is shown in Figure 8.

With the increase of the reliability of inbound gate, the expected average travel time gradually decrease and remain stable after the reliability reaches 0.6. Under the current passenger flow conditions, the reliability of the inbound gate needs to be maintained at 0.7 or higher. Similarly, the reliability of the outbound gate and escalator needs to be maintained at 0.8 or higher. When key equipment does not reach the specified reliability, appropriate maintenance measures need to be taken to maintain station capacity.
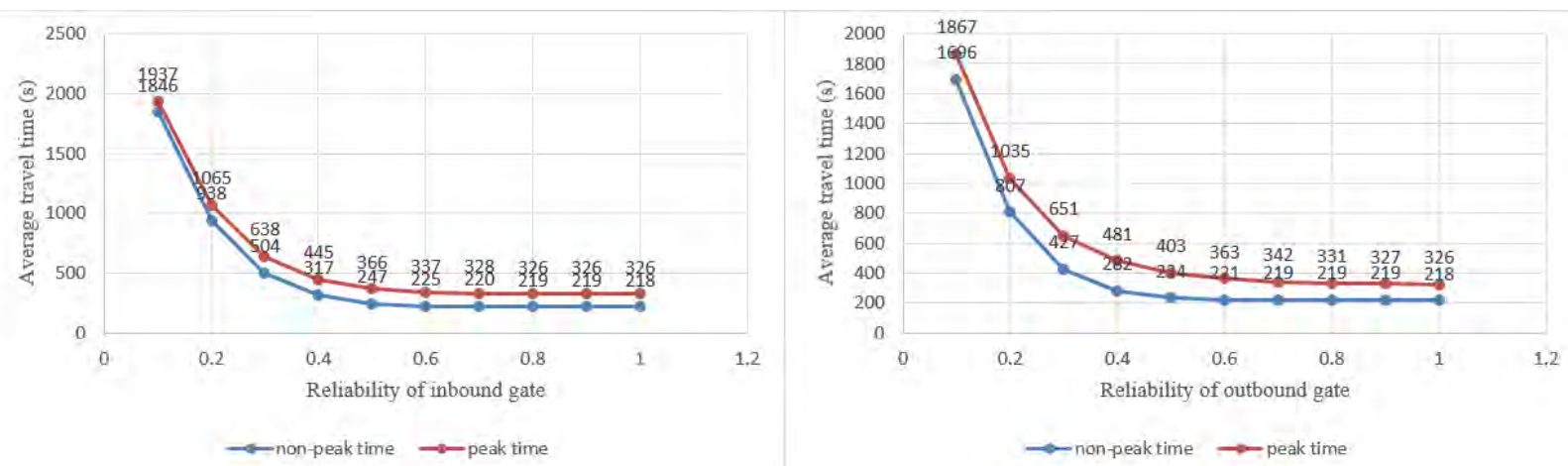

Fig 7. The impact of the reliability of gate on average travel time.

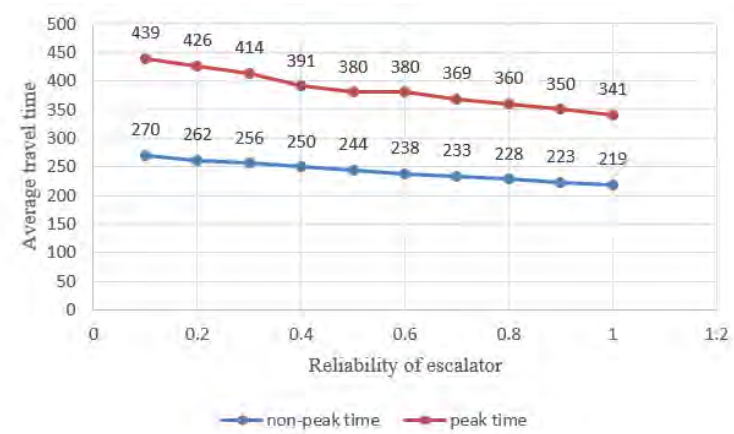

Fig 8 . The impact of the reliability of escalator on average travel time.

\section{Summary}

In this paper, the key equipment capacity model and station capacity model considering key equipment failure are established using the idea of binomial distribution and mathematical expectation and this paper analyzes the impact of key equipment failures on the average travel time of passengers on the base of AnyLogic simulation model, and gives corresponding maintenance recommendations. There are some directions possible with this research. For example, failure data of key equipment can be analyzed to get the corresponding reliability distribution to improve subway station capacity model considering key equipment failures.

\section{Acknowledegments}

This work is partly supported by Chinese Nation Key Projece of Research and Development (Contract No. 2017YFB1201203-001). 


\section{References}

[1]. Qi Zhang, Baoming Han. Modeling and simulation of passenger alighting and boarding movement in Beijing metro stations. Transportation Research Part C, 2008, 16 (5), 635-649.

[2]. Xu X Y, Liu J, Li H Y, et al. Analysis of subway station capacity with the use of queueing theory. Transportation Research Part C, 2014, 38(1); 28-43.

[3]. Feng Chen, Qi Wu, et al. Relationship analysis on station capacity and passenger flow: a case of Beijing subway line 1. Journal of Transportation Systems Engineering and Information Technology, 2009, 9 (2), 93-98.

[4]. Cruz, F.R.B., Smith, et al. Approximate analysis of M/G/c/c state-dependent queueing networks. Computers \& Operations Research, 2007, 34 (8), 2332-2344.

[5]. Shouhua Cao, Zhenzhou Yuan. Characteristics analysis and model establishment for passenger boarding time in urban rail transit. Journal of the China Railway Society, 2009, 31 (3), 89-93. 